\title{
Complications Chroniques de la Drépanocytose dans une Population de 893 Patients Adultes Suivis au CHU Campus de Lomé
}

\author{
Kueviakoe MDI., \\ Service d'hématologie, CHU Sylvanus Olympio, \\ Faculté des Sciences de la Santé/Université de Lomé, Togo \\ Padaro E., \\ Service d'hématologie, CHU Campus de Lomé, \\ Faculté des Sciences de la Santé/Université de Lomé, Togo \\ Magnang $\boldsymbol{H}$., \\ Centre National de Recherche et de Soins aux Drépanocytaires, \\ Faculté des Sciences de la Santé/Université de Lomé, Togo \\ Mawussi K., \\ Service d'hématologie, \\ Faculté des Sciences de la Santé/Université de Kara, Togo

$$
\begin{gathered}
\text { Womey KMC., } \\
\text { Layibo Y., } \\
\text { Agbétiafa K., }
\end{gathered}
$$ \\ Service d'hématologie, \\ Faculté des Sciences de la Santé/Université de Lomé, Togo \\ Koudokpo ADN., \\ Service d'hématologie, CHU Campus de Lomé, Togo \\ Vovor A., \\ Service d'hématologie, CHU Sylvanus Olympio, \\ Faculté des Sciences de la Santé/Université de Lomé, Togo
}

Doi: 10.19044/esj.2019.v15n12p318 URL:http://dx.doi.org/10.19044/esj.2019.v15n12p318

\section{Résumé}

Objectif : Décrire les fréquences des complications chroniques de la drépanocytose chez le sujet adulte SS et SC suivis au CHU Campus de Lomé. Patients et méthodes : Il s'agit d'une étude descriptive portant sur les complications chroniques de la drépanocytose chez 893 patients drépanocytaires SS ou SC âgés d'au moins 18 ans et suivis entre 2000 et 2017. Les données sociodémographiques, cliniques et évolutives (complications chroniques) ont été collectées des dossiers. Le traitement statistique et l'analyse des données ont été réalisés à l'aide du logiciel $\mathrm{r}$ studio 3.3.2. 
Résultats : La sex-ratio H/F était de 0,81. L'âge moyen des patients était $31,81 \pm 10,82$ ans. La tranche d'âge de 18 à 27 ans était la plus représentée (43,90\%). Selon le phénotype, 46,58\% étaient drépanocytaires SS et 53,42\% drépanocytaires hétérozygotes SC. Au moins une complication était présente chez $42,55 \%$ de nos patients. Cette fréquence était de 31,97\% chez les SS et de $51,78 \%$ chez les SC. Les complications ischémiques étaient présentes chez $34,15 \%$ de l'ensemble de nos patients. Elles représentaient 36,78\% des complications chroniques chez les SS et $31,87 \mathrm{chez}$ les SC : la rétinopathie drépanocytaire $(17,25 \%)$ et l'ostéonécrose aseptique (14\%) étaient les plus fréquentes, suivies de l'atrophie splénique (4,59\%), l'ulcère cutané $(3,25 \%)$, les AVC $(1,23 \%)$ et les complications rénales $(1,12 \%)$. Les complications hémolytiques étaient présentes chez $14,89 \%$ des patients et étaient statistiquement liées au phénotype SS. Parmi elles, la lithiase biliaire était la plus fréquente $(10,53 \%)$, suivie de la cardiopathie drépanocytaire $(1,57 \%)$ et de l'hypertension artérielle pulmonaire $(0,22 \%)$. Conclusion : Les complications chroniques du drépanocytaire adulte demeurent fréquentes en milieu hospitalier au Togo. Des études sur les facteurs prédictifs du développement de ces complications s'avèrent nécessaires.

Mots-clés : Drépanocytose, Complications chroniques, Togo 


\title{
Chronic Complications of Sickle Cell Disease in a Population of 893 Adult Patients Followed at Campus Teaching Hospital of Lomé
}

\author{
Kueviakoe MDI., \\ Service d'hématologie, CHU Sylvanus Olympio, \\ Faculté des Sciences de la Santé/Université de Lomé, Togo \\ Padaro E., \\ Service d'hématologie, CHU Campus de Lomé, \\ Faculté des Sciences de la Santé/Université de Lomé, Togo \\ Magnang $\boldsymbol{H}$., \\ Centre National de Recherche et de Soins aux Drépanocytaires, \\ Faculté des Sciences de la Santé/Université de Lomé, Togo \\ Mawussi K., \\ Service d'hématologie, \\ Faculté des Sciences de la Santé/Université de Kara, Togo

$$
\begin{gathered}
\text { Womey KMC., } \\
\text { Layibo Y., } \\
\text { Agbétiafa K., }
\end{gathered}
$$ \\ Service d'hématologie, \\ Faculté des Sciences de la Santé/Université de Lomé, Togo \\ Koudokpo ADN., \\ Service d'hématologie, CHU Campus de Lomé, Togo \\ Vovor A., \\ Service d'hématologie, CHU Sylvanus Olympio, \\ Faculté des Sciences de la Santé/Université de Lomé, Togo
}

\begin{abstract}
Objective: To describe chronic complications frequencie of sickle cell disease in adult SS and SC patients followed at Campus Teaching Hospital of Lomé. Patients and methods: This was a descriptive study of the chronic complications of sickle cell disease in 893 patients, SS and SC at least 18 years of age and followed between 2000 and 2017. Sociodemographic, clinical and progressive data (chronic complications) were collected from records. Statistical processing and data analysis were performed using the software $r$ studio 3.3.2. Results: The sex ratio $\mathrm{H} / \mathrm{F}$ was 0,81 . The mean age of the
\end{abstract}


patients was $31,81 \pm 10.82$ years. The age group 18 to 27 was the most represented (43.90\%). According to the phenotype, $46.58 \%$ were sickle cell SS and $53.42 \%$ sickle cell heterozygous SC. At least one complication was present in $42.55 \%$ of our patients. This frequency was $31,97 \%$ for SS and $51,78 \%$ for SC. Ischemic complications were present in $34.15 \%$ of all our patients. They accounted for $36.78 \%$ of chronic complications among SS and 31.87 in SC: sickle cell retinopathy (17.25\%) and aseptic osteonecrosis (14\%) were the most common, followed by splenic atrophy $(4.59 \%)$, cutaneous ulcer ( $3.25 \%)$, stroke (1.23\%) and renal complications (1.12\%). Hemolytic complications were present in $14.89 \%$ of patients and were statistically related to the SS phenotype. Among them, cholelithiasis was the most common $(10.53 \%)$, followed by sickle cell heart disease $(1.57 \%)$ and pulmonary arterial hypertension (0.22\%). Conclusion: Chronic complications of adult sickle cell disease remain frequent in hospitals in Togo. Studies on the predictive factors for the development of these complications are necessary.

Keywords: Sickle cell disease, Chronic complications, Togo

\section{Introduction}

La drépanocytose est une anomalie qualitative de l'hémoglobine caractérisée par une mutation du $6^{\mathrm{e}}$ acide aminé de la chaîne bêta de globine où l'acide glutamique est remplacé par la valine. Cette mutation aboutit à la formation de l'hémoglobine $\mathrm{S}(\mathrm{HbS})$ dont la propriété essentielle est la gélification, responsable de la falciformation. C'est la pathologie génétique la plus fréquente au monde avec plus de 120 millions d'individus atteints $\mathrm{La}$ distribution géographique de la maladie s'observe majoritairement en Afrique (Rees DC et al, 2010). Au Togo, selon une étude hospitalière, la prévalence de la drépanocytose homozygote SS est de 1,3\% et celle de la drépanocytose hétérozygote composite SC, de 2,6\% de la population (Segbena AY et al, 2002).

L'histoire naturelle de la drépanocytose homozygote est émaillée de complications aigues et chroniques dont la distribution est variable selon l'âge (Dokekias AE et al, 2001). Les complications aiguës faites de crises douloureuses intenses, de crises de déglobulisation et d'infections sont les plus fréquentes chez l'enfant. Chez l'adulte, en dehors des complications aigues, ce sont les complications chroniques qui dominent le tableau clinique. Ces complications chroniques résultent d'une atteinte irréversible et dégénérative de plusieurs organes dont l'os, le rein, l'œil, le poumon, le cerveau. (Dokekias $\mathrm{AE}$ et al, 2001). Elles découlent de deux (02) mécanismes que sont l'hémolyse chronique et l'ischémie. Les complications hémolytiques regroupent l'hypertension artérielle pulmonaire (HTAP), la lithiase biliaire, la cardiopathie drépanocytaire. Les complications ischémiques comprennent 
l'ulcère cutané, l'ostéonécrose aseptique, l'accident vasculaire cérébral, la rétinopathie drépanocytaire et les complications rénales. Toutefois il faut préciser que cette classification n'est pas exhaustive. En effet, les deux mécanismes sont bien souvent intriqués dans la physiopathologie de ces complications. Un suivi régulier et un bilan annuel sont recommandés pour dépister et traiter précocement ces complications spécifiques de la maladie (HAS, 2010). Le contenu de ce bilan varie selon l'âge et le contexte clinique. Les progrès notés dans la prise en charge du drépanocytaire enfant et adolescent ont augmenté l'espérance de vie de ceux-ci et par conséquent accru le nombre de sujets drépanocytaires adultes (Wierenga KJ et al, 2001).

A notre connaissance, aucune étude togolaise n'a permis d'évaluer la répartition des différentes complications chroniques de la maladie chez le sujet adulte. Nous avons donc entrepris ce travail pour décrire la fréquence des complications chroniques de la drépanocytose SS et SC chez les sujets adultes suivis au CHU Campus de Lomé de 2000 à 2017.

\section{Methodologie}

Il s'agit d'une étude descriptive réalisée dans le service d'hématologie du Centre Hospitalier Universitaire (CHU) Campus de Lomé et a concerné les drépanocytaires adultes SS ou SC suivis entre janvier 2000 et décembre 2017. Ont été inclus, les patients âgés d'au moins 18ans le $1^{\text {er }}$ janvier 2018, régulièrement suivis pendant au moins une année (ayant fait au moins 3 consultations) et dont l'électrophorèse de l'hémoglobine est compatible avec une drépanocytose majeure SS ou SC. Chaque patient suivi dans le service dispose d'un dossier médical qui comprend une observation clinique initiale détaillée (faite le jour de l'ouverture du dossier) qui est mise à jour à l'occasion de chaque consultation de suivi ou au décours d'une complication intercurrente et les résultats des bilans biologiques et morphologiques réalisés à titre d'évaluation ou orientés selon la symptomatologie clinique. Les patients qui n'ont pas été inclus dans cette étude sont ceux qui ont fait moins de 3 consultations, les patients perdus de vue et les patients ayant moins de 18 ans au $1^{\text {er }}$ janvier 2018.

Le traitement statistique et l'analyse des données ont été réalisés à l'aide du logiciel $\mathrm{R}$ studio 3.3.2. Les différentes fréquences sont présentées avec leur intervalle de confiance à $95 \%$. Les variables qualitatives sont présentées sous forme d'effectifs et de proportion et les variables quantitatives sous forme d'étendue, de moyenne avec écart type ou sous forme de médiane avec intervalle interquartile. Les tests de chi 2 ou de Fischer ont servi à l'analyse comparative selon l'effectif théorique. Les tests statistiques ont été réalisés avec un seuil significatif $\mathrm{p}=0,05$. 


\section{Resultats}

\section{Données épidémiologiques}

De janvier 2000 à décembre 2017, le service d'hématologie clinique du CHU Campus a accueilli 4412 patients dont 371 pour une hémopathie maligne et 3807 drépanocytaires tout âge confondu. Parmi les drépanocytaires, 1036 dossiers de patients supérieurs à 18 ans ont été dépouillés et 893 ont été retenus.

\section{Age et sexe}

Notre échantillon était constitué de 400 (44,79\%) hommes et 493 $(55,21 \%)$ femmes, soit une sex-ratio H/F de 0,81 . L'âge moyen des patients

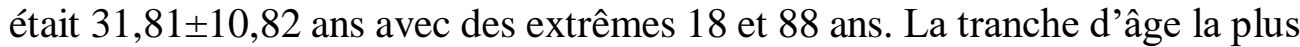
représentée était celle des 18-27 ans (figure 1). L'âge moyen des patients de sexe masculin était $31,29 \pm 11,12$ ans et celui des patients de sexe féminin de $32,23 \pm 10,55$ ans. Les patients ayant au moins une complication avaient un âge moyen de $31,75 \pm 9,76$ ans pour les sujets SS et de 38,42 $\pm 11,96$ ans pour les $\mathrm{SC}$ ( $\mathrm{p}$ value $<0,0001)$.

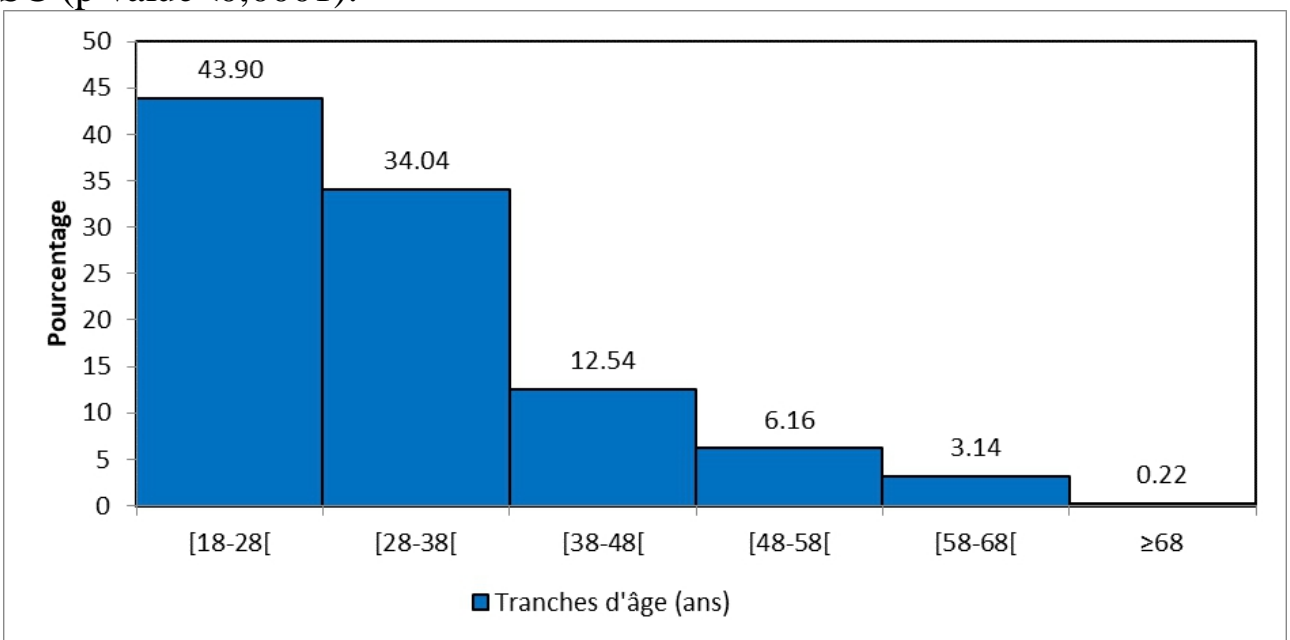

Figure 1 : Répartition des patients par tranches d'âge de 10 :

\section{Données cliniques}

Selon le phénotype, 416 drépanocytaires (46,58\%) étaient homozygotes SS et $477(53,42 \%)$ hétérozygotes SC.

\section{Fréquence globale des complications}

Au moins une complication était présente chez 42,55\% de nos patients. Cette fréquence était de $31,97 \%$ chez les SS et de $51,78 \%$ chez les SC (p $<0,0001)$ (tableau I). 
Tableau I: Fréquence globale des complications chroniques

\begin{tabular}{llll}
\hline & Effectifs n(N) & Pourcentage \% & IC 95\% \\
Tous les patients & $380(893)$ & 42,55 & $39,29-45,88$ \\
Patients SS & $133(416)$ & $31,97 \% *$ & $27,56-36,72 \%$ \\
Patients SC & $247(477)$ & $51,78 \% *$ & $47,27-56,06 \%$ \\
\hline \multicolumn{2}{c}{$*$ p $<0,0001$ (test de chi2 d'indépendance), n=effectif des patients présentant des } \\
\multicolumn{4}{c}{ complications, $\mathrm{N}=$ effectif de l'échantillon }
\end{tabular}

Répartition nosologique des complications chroniques

La rétinopathie drépanocytaire et l'ostéonécrose étaient les complications les plus fréquentes avec respectivement $17,25 \%$ et $14 \%$ (tableau II).

Tableau II: Fréquence des différentes complications



*=Test de chi-2 d'indépendance $; * *=$ Test exact de Fisher

Fréquence des deux groupes de complications chroniques selon le phénotype

Les complications ischémiques étaient les plus fréquentes avec une fréquence globale de $34,15 \%$ contre $14,89 \%$ de complications hémolytiques. (Tableau III). 
Tableau III: Fréquence des deux types de complications chroniques selon le phénotype

\begin{tabular}{llllllll}
\hline & \multicolumn{2}{l}{$\begin{array}{l}\text { Ensemble } \\
(\mathrm{N}=893)\end{array}$} & & \multicolumn{2}{c}{$\begin{array}{l}\mathrm{SS} \\
(\mathrm{N}=416)\end{array}$} & \multicolumn{2}{c}{$\mathrm{SC}$} \\
& $\mathrm{n}$ & $\%$ & $\mathrm{n}$ & $\%$ & $\mathrm{n}$ & $\%$ & $\mathrm{p}$ value \\
& 133 & 14,89 & 94 & 22,60 & 39 & 8,18 & $<0,0001^{*}$ \\
Hémolytique & & & & & & \\
Ischémique & 305 & 34,15 & 153 & 36,78 & 152 & 31,87 & $0,1225^{*}$ \\
\hline \multicolumn{7}{c}{$*=$ Test de chi-2 d'indépendance, $\mathrm{n}=$ effectif } &
\end{tabular}

\section{Complications hémolytiques}

Les complications hémolytiques étaient statistiquement plus fréquentes chez les sujets SS comparativement au sujet SC (tableau IV).

Tableau IV : Répartition des différentes complications hémolytiques

\begin{tabular}{|c|c|c|c|c|c|c|c|}
\hline & \multicolumn{3}{|c|}{$\begin{array}{l}\text { Ensemble } \\
(\mathrm{N}=133)\end{array}$} & \multicolumn{2}{|c|}{$\begin{array}{l}\mathrm{SS} \\
(\mathrm{N}=94)\end{array}$} & \multirow{2}{*}{$\begin{array}{l}\mathrm{SC} \\
(\mathrm{N}=39) \\
\%\end{array}$} & \multirow[t]{2}{*}{$p$ value } \\
\hline & $\mathrm{n}$ & $\%$ & $\mathrm{n}$ & $\%$ & $\mathrm{n}$ & & \\
\hline HTAP & 2 & 1,50 & 2 & 2,13 & 0 & 0,00 & $0,9999 * *$ \\
\hline Lithiase biliaire & 94 & 70,68 & 89 & 94,68 & 5 & 12,82 & $<0,0001^{*}$ \\
\hline $\begin{array}{l}\text { Cardiopathie } \\
\text { drépanocytaire }\end{array}$ & 14 & 10,53 & 8 & 8,51 & 6 & 15,38 & $0,4247 *$ \\
\hline
\end{tabular}

La lithiase biliaire était la plus fréquente des complications hémolytiques de notre série. Sa survenue était liée au phénotype SS. Elle était diagnostiquée plus précocement chez le patient SS que chez le sujet SC avec un âge moyen au diagnostic 25,92 ans contre 32,23 ans ( $\mathrm{p}=0,0041)$. La cardiopathie drépanocytaire représentait la deuxième complication hémolytique la plus retrouvée avec $10,53 \%$ et $1,92 \%$ des patients SS et $1,26 \%$ des patients SC $(\mathrm{p}=0,4247)$, avaient une cardiopathie drépanocytaire.

\section{Complications ischémiques}

La rétinopathie drépanocytaire et l'ostéonécrose aseptique sont les complications les plus fréquentes. L'AVC a été retrouvé chez 3,61\% des patients atteints d'une complication ischémique (tableau V).

Tableau V: Répartition des différentes complications ischémiques selon le phénotype

\begin{tabular}{|c|c|c|c|c|c|c|c|}
\hline & \multicolumn{3}{|c|}{$\begin{array}{l}\text { Ensemble } \\
(\mathrm{N}=305)\end{array}$} & \multicolumn{2}{|c|}{$\begin{array}{l}\text { SS } \\
(\mathrm{N}=153)\end{array}$} & \multirow{2}{*}{$\begin{array}{l}\begin{array}{l}\mathrm{SC} \\
(\mathrm{N}=152)\end{array} \\
\%\end{array}$} & \multirow[t]{2}{*}{$\mathrm{p}$ value } \\
\hline & $\mathrm{n}$ & $\%$ & $\mathrm{n}$ & $\%$ & $\mathrm{n}$ & & \\
\hline Ulcère cutanée & 29 & 9,51 & 27 & 17,65 & 2 & 1,32 & $<0,0001 *$ \\
\hline \multirow{2}{*}{$\begin{array}{l}\text { Ostéonécrose } \\
\text { aseptique }\end{array}$} & 125 & 40,98 & 57 & 37,26 & 68 & 44,74 & $0,1840^{*}$ \\
\hline & 11 & 3,61 & 10 & 6,54 & 1 & 0,66 & $0,0059 *$ \\
\hline
\end{tabular}




\begin{tabular}{llllllll}
\hline $\begin{array}{l}\text { Complications } \\
\text { cérébrales }\end{array}$ & & & & & & & \\
$\begin{array}{l}\text { Rétinopathie } \\
\text { drépanocytaire }\end{array}$ & 154 & 50,49 & 61 & 39,87 & 93 & 61,18 & $0,0002^{*}$ \\
$\begin{array}{l}\text { Complications } \\
\text { rénales }\end{array}$ & 10 & 3,28 & 5 & 3,27 & 5 & 3,22 & $0,9916^{*}$ \\
$\begin{array}{l}\text { Atrophie } \\
\text { splénique }\end{array}$ & 41 & 13,44 & 37 & 24,18 & 4 & 2,63 & $<0,0001^{*}$ \\
\hline & $*=T e s t$ de chi-2 d'indépendance
\end{tabular}

\section{Discussion}

\section{Limites}

Les principales limites de cette étude étaient liées à son caractère rétrospectif: incomplétude des dossiers, défaut de précision, données manquantes. Cette étude ne nous a pas permis également de déterminer avec précision l'âge réel de survenue des différentes complications. Une étude de cohorte prospective portant sur des drépanocytaires dépistés et suivi dès la naissance permettra de mieux appréhender cet aspect. Les difficultés étaient relatives au nombre important de dossiers exploités manuellement et aux données manquantes liées à la tenue des dossiers médicaux. Vu le nombre important de drépanocytaires suivis au CHU Campus, une informatisation des dossiers des patients permettrait de palier à ces insuffisances et aux problèmes d'archivages. Néanmoins, cette étude a le mérite de permettre de faire la cartographie actuelle des complications chroniques chez l'adulte drépanocytaire.

\section{Age et sexe}

La sex-ratio de 0,81 était superposable à celle de Seck et al au Sénégal (2017) et Elira Dokekias et al au Congo (2001). Par contre, Tolo et al en Côte d'Ivoire (2010) avait retrouvé une sex-ratio à 1,28. L'âge moyen de $31,81 \pm 10,82$ ans de notre série était relativement plus élevé car notre échantillon ne prenait en compte que les drépanocytaires adultes âgés de 18 ans et plus. L'âge moyen des patients SS ayant au moins une complication est comparable à celui de Tolo et al en Côte d'Ivoire (2010) qui était de 26,1 ans.

\section{Fréquence}

Il existe une différence statistiquement significative de la fréquence globale des complications chroniques entre les SS et les SC, ces complications étant plus fréquentes chez les SC. Ces résultats sont comparables à ceux de Seck et al (2017) avec des fréquences de $26,53 \%$ et $18,36 \%(p=0,0001)$ respectivement. Cependant cette fréquence est différemment appréciée selon 
les séries. Des fréquences de $55,7 \%$ et $48 \%$ ont été rapportées respectivement au Congo et aux USA (Dokekias AE et al, 2001 ; Powars DR et al, 2005). Dans l'étude réalisée aux USA, Powars et al (2005) avaient rapporté que $73 \%$ des patients décédés avaient au moins une complication chronique. La plupart des études sous-estiment la fréquence réelle de ces complications chroniques $\mathrm{du}$ fait de leur caractère souvent asymptomatique mais aussi de la non systématisation de leur dépistage pour des raisons de coût et d'accessibilité. Ainsi, Manci et al (2003) rapportait aux USA une fréquence de 74,7 \% de complications chroniques à l'autopsie des patients drépanocytaires homozygotes décédés contre seulement 25,3\% de complications dépistées sur des arguments cliniques et paracliniques lors de leur vie.

\section{Complications hémolytiques}

Dans notre étude, la survenue des complications hémolytiques était statistiquement liée au phénotype SS. Plusieurs études accordent en effet une place prédominante aux complications hémolytiques chez le sujet drépanocytaire homozygote (Dokekias AE et al, 2001 ; Tolo et al, 2010 ; Diop $\mathrm{S}$ et al 2010). La fréquence plus élevée des complications hémolytiques chez les sujets SS comparativement aux sujets SC est expliquée par l'importance de l'hémolyse chronique chez les patients homozygotes.

La lithiase biliaire était la plus fréquente des complications hémolytiques de notre série. Sa survenue était liée au phénotype SS. Elle était diagnostiquée plus précocement chez le patient SS que chez le sujet SC avec un âge moyen au diagnostic 25,92 ans contre 32,23 ans ( $\mathrm{p}=0,0041)$. Ngolet et al (2018) avaient retrouvé également une prédominance de la lithiase $(40,16 \%)$ dans une étude portant sur des patients SS de plus de 18 ans. La lithiase biliaire restant asymptomatique dans la grande majorité des cas (Payen J-L et, 2011), la faible fréquence retrouvée dans notre étude pourrait être expliquée par la non réalisation systématique de l'échographie abdominale dans le suivi des patients drépanocytaires. Cette dernière n'est souvent réalisée que devant un signe d'appel alors qu'elle devrait être systématique chaque année à partir de l'âge de 03 ans ou lors de signe évocateurs (HAS, 2010). Dans une étude cas-témoin réalisée au Sénégal, Seck et al (2017) par contre avaient retrouvé une même répartition de la lithiase biliaire dans les deux phénotypes.

La cardiopathie drépanocytaire représentait la deuxième complication hémolytique la plus retrouvée avec 10,53\%. Pour Tolo et al (2010) en revanche, les atteintes cardiaques représentaient la première complication hémolytique avec 20,8\%. Dans notre étude, 1,92\% des patients SS et 1,26\% des patients SC $(\mathrm{p}=0,4247)$, avaient une cardiopathie drépanocytaire. L'hémolyse plus accrue dans le phénotype SS, explique la prédominance des cardiopathies chez les patients homozygotes SS. Nos résultats rejoignent ceux 
de Seck et al (2017) avec 6,12\% des SS comparativement à 0\% SC (p=0,004). La faible fréquence des atteintes cardiaques retrouvée dans notre étude pourrait être expliquée par la non réalisation systématique au cours du suivi des sujets drépanocytaires des bilans de dépistage des cardiopathies drépanocytaires (radiographie du thorax, ECG, échodoppler cardiaque). Pour l'échodoppler cardiaque par exemple, la recommandation est de la faire chaque année chez les patients de plus de 06 ans (HAS 2010). De plus les signes d'appel (dyspnée, douleur thoracique) ne sont pas directement rattachés à la cardiopathie drépanocytaire mais plutôt au terrain drépanocytaire, ce qui conduit à un sous-diagnostic par manque d'exploration appropriée.

La fréquence de l'hypertension artérielle pulmonaire dans notre série était inférieure à celles rapportées dans des études antérieures (Ngolet et al, 2018 ; Gladwin et al 2004). En utilisant également l'échodoppler cardiaque comme outil diagnostic, Ngolet et al (2018) ont retrouvé une fréquence de $30,33 \%$ chez des patients homozygotes de plus de 18 ans. Gladwin et al (2004) quant à eux ont rapporté une fréquence de $32 \%$ dans une population de drépanocytaire dont l'âge moyen était de 36 ans.

\section{Complications ischémiques}

Elles étaient présentes chez 34,15\% de nos patients. Les plus fréquentes étaient la rétinopathie drépanocytaire et l'ostéonécrose de la tête fémorale avec respectivement $17,25 \%$ et $14,00 \%$. Tolo et coll (2010) ont rapporté également une prédominance de ces deux types de complications ischémiques.

La fréquence de la rétinopathie drépanocytaire de notre série était proche de celle de Condon et coll en Jamaïque (1975) 17,5\%. Cependant, nos résultats sont inférieurs à ceux de Diallo au Burkina-Faso $(49,4 \%)$ et de Balo au Togo (59,57\%) (Diallo et al, 2009 ; Balo et al, 1996). La différence des moyennes d'âge, du mode de recrutement des patients et des méthodes diagnostiques pourrait expliquer en partie cette disparité.

L'ostéonécrose aseptique représentait la deuxième complication ischémique la plus fréquente (40,98\% des complications ischémiques). Par contre, elle était la seule complication ischémique retrouvée par Ngolet et coll (2017) dans une étude portant sur les drépanocytaires homozygotes de plus de 18 ans.

Le risque vasculaire cérébral est une menace réelle qui pèse sur les patients drépanocytaires essentiellement $\mathrm{SS}$ et ce de manière précoce (Kossorotoff $\mathrm{M}$ et al, 2014). Dans notre étude, elle représentait 3,61\% des complications ischémiques. Sa fréquence était liée au type de drépanocytose. Les mêmes résultats ont été rapportés par Ohene-Frempong et al (1998) avec une fréquence de $4,07 \%$ chez les sujets SS et $0,80 \%$ chez les SC. 
Il y a une forte probabilité que les fréquences des complications cérébrales en général et surtout des infarctus silencieux soient sous-estimés dans notre étude. En effet, le scanner cérébral n'est le plus souvent réalisé que devant des signes d'appel. Et dans la majorité des cas, il révèle des lésions déjà constituées. Les conséquences motrices et cognitives de ces accidents peuvent être dramatiques Il est donc important de mettre en place très précocement dans l'enfance, des stratégies de dépistage des patients à risque et de prévention de survenue des AVC (par doppler transcrânien) afin d'instaurer les thérapeutiques appropriés pour les patients à risque.

L'ulcère cutané était présent chez 3,25\% des patients de notre étude et représentait $9,51 \%$ des complications ischémiques. Nos résultats sont similaires à ceux de Powars et al (2002) avec 14,4\% contre 2,8\%. Ces résultats confortent ceux de Minniti et al (2014) qui ont démontré que l'ulcère cutané était plus consécutif à une hémolyse chronique et à une vasculopathie qu'à une ischémie.

Les complications rénales étaient présentes chez 1,12\% des patients de notre série et représentaient $3,28 \%$ des complications ischémiques. Nos résultats sont très inférieurs à ceux de Zadeii aux USA qui a retrouvé une fréquence de 30 à $40 \%$ pour la nécrose papillaire chez des patients homozygotes (Zadeii $\mathrm{G}$ et al, 1997). Cette faible fréquence dans notre étude peut être expliquée par un manque de dépistage systématique de ces complications notamment par la recherche d'une microalbuminurie.

L'atrophie splénique était présente chez $4,59 \%$ des patients de notre étude et représentait $13,44 \%$ des complications ischémiques. Elle était statistiquement plus fréquente chez les patients SS que chez les patients SC. Nos résultats sont inférieurs à ceux de Tolo et al (2010) qui ont trouvé une fréquence de 37,5\%. Selon Beauvais (1982), la rate étant le siège d'infarctus récidivants, elle s'atrophierait progressivement. Elle survient chez le drépanocytaire homozygote SS vers l'âge de six ans, et succède à la phase congestive des premières années (Beuzard Y et al, 1992). L'asplénie qui en résulte est à la base de la susceptibilité aux infections du sujet drépanocytaire.

\section{Conclusion}

Les complications chroniques demeurent fréquentes chez le sujet drépanocytaire adulte au Togo malgré la mise en place d'un suivi régulier. Elles sont plus fréquentes chez les sujets SC mais apparaissent plus précocement chez les sujets SS. L'ostéonécrose aseptique et la rétinopathie drépanocytaire étaient les complications ischémiques les plus retrouvées et la lithiase biliaire était la complication hémolytique la plus fréquente. Il est nécessaire d'organiser un dépistage plus précoce des complications chroniques et d'initier des études sur les facteurs prédictifs de leur survenue. Une étude de cohorte dès la naissance permettra d'appréhender de façon plus 
précise, l'âge de survenue de ces complications afin de mieux organiser les bilans de dépistage. Une prise en charge plus adaptée et complète impliquant les différents acteurs concernés doit également être mis en place en vue de réduire l'incidence de ces complications chroniques. Cela permettra d'améliorer le mode de vie des patients, d'augmenter leur espérance de vie. L'ouverture récente du centre national de recherche et de soins aux drépanocytaires est une note d'espérance.

\section{References:}

1. Rees DC, Williams TN, Gladwin MT (2010). Sickle-cell disease. The Lancet; 376: 2018-31.

2. Ségbéna AY, Kueviakoe IMD, Messie AK, Napo-Koura IG, Vovor A, David M (2002). Les anomalies de l'hémoglobine au Centre Hospitalier Universitaire de Lomé, Togo. Med Trop; 62: 51-54.

3. Dokekias AE, Nzingoula S (2001). Profil du sujet drépanocytaire homozygote après l'âge de 30 ans. Médecine D'Afrique Noire; 48: 10.

4. Haute Autorité de Santé (2010). Prise en charge de la drépanocytose chez l'enfant et l'adolescent. Saint-Denis La Plaine.

5. Wierenga KJ, Hambleton IR, Lewis NA, Unit SC (2001). Survival estimates for patients with homozygous sickle-cell disease in Jamaica: a clinic-based population study. The Lancet; 357 :680-3.

6. Seck M, Faye BF, Sall A, Fall A, Touré SA, Dieng N, et al (2017). Profil course of SC sickle-cell patients in Dakar: a case-control study with SS sickle cell form. Mali Med; 32: 7-12.

7. Tolo-Diebkilé A, Koffi KG, Nanho DC, Sawadogo D, Kouakou B, Siransy-Bogui L, et al (2010). Drépanocytose homozygote chez l'adulte ivoirien de plus de 21 ans. Cah Santé; 2: 63-67.

8. Powars DR, Chan LS, Hiti A, Ramicone E, Johnson C (2005). Outcome of Sickle Cell Anemia: A 4-Decade Observational Study of 1056 Patients. Medicine (Baltimore); 84: 363.

9. Manci EA, Culberson DE, Yang Y-M, Gardner TM, Powell R, Haynes Jr J, et al (2003). Causes of death in sickle cell disease: an autopsy study. Br J Haematol; 123: 359-365.

10. Diop S, Diop D, Seck M, Guèye Y, Faye A, Dièye TN, et al (2010). Facteurs prédictifs des complications chroniques de la drépanocytose homozygote chez l'adulte à Dakar (Sénégal). Médecine Trop; 70: 471.

11. Ngolet L, Ova JON, Ntsiba H, Dokekias AE (2018). Les complications chroniques du sujet drépanocytaire adulte à Brazzaville. Health Sci Dis;18: disponible sur: http://www.hsdfmsb.org/index.php/hsd/article/view/812.

12. Payen J-L, Muscari F, Vibert É, Ernst O, Pelletier G (2011). Lithiase biliaire. Presse Médicale; 40: 567-580. 
13. Gladwin MT, Sachdev V, Jison ML, Shizukuda Y, Plehn JF, Minter $\mathrm{K}$, et al (2004). Pulmonary hypertension as a risk factor for death in patients with sickle cell disease. N Engl J Med; 350: 886- 95.

14. Condon PI, Serjeant GR (1975). The progression of sickle cell eye disease in Jamaica. Doc Ophthalmol; 39: 203-10.

15. Diallo JW, Sanfo O, Blot I, Meda N, Sawadogo P, Ouedraogo A, et al (2009). Étude épidémiologique et facteurs pronostiques de la rétinopathie drépanocytaire à Ouagadougou (Burkina Faso). J Fr Ophtalmol; 32: 496-500.

16. Balo KP, Segbena K, Mensah A, Mihluedo H, Bechetoille A (1996). Hemoglobinopathies and retinopathies in Lomé UHC. J Fr Ophtalmol; 19: 497-504.

17. Kossorotoff M, Grevent D, de Montalembert M (2014). Drépanocytose et atteinte vasculaire cérébrale chez l'enfant. Arch Pédiatrie; 21: 404-14.

18. Ohene-Frempong K, Weiner SJ, Sleeper LA, Miller ST, Embury S, Moohr JW, et al (1998). Cerebrovascular Accidents in Sickle Cell Disease: Rates and Risk Factors. Blood; 91: 288-94.

19. Powars DR, Hiti A, Ramicone E, Johnson C, Chan L (2002). Outcome in hemoglobin SC disease: A four-decade observational study of clinical, hematologic, and genetic factors. Am J Hematol; 70: 206-15.

20. Minniti CP, Delaney K-MH, Gorbach AM, Xu D, Lee C-CR, Malik N, et al (2014). Vasculopathy, inflammation, and blood flow in leg ulcers of patients with sickle cell anemia. Am J Hematol; 89: 1-6.

21. Zadeii G, Lohr JW (1997). Renal papillary necrosis in a patient with sickle cell trait. J Am Soc Nephrol; 8: 1034-9.

22. Beauvais P (1982). Drépanocytose et asplénie fonctionnelle. Arch Fr Pédiatr; 39: 141-144.

23. Beuzard Y, Galacteros F (1992). Drépanocytose. In: Hématologie. Dreyfus et coll. Flammarion, Méd Science 3ème Edition, 378-392. 